\title{
Fever of unknown origin - Hidden in the head
}

\author{
Kamal Kishore Pandita, Khalid Javid Bhat, Sushil Razdan, Rattan Parkash Kudyar \\ Department of Internal Medicine, ASCOMS \& Hospitals, Jammu \& Kashmir, India
}

\begin{abstract}
The original algorithmic approach, as outlined by de Kleijn and colleagues and practiced commonly, envisages performing computed tomography (CT) of chest, abdomen, and pelvis in patients with classical fever of unknown origin (FUO), in whom no potentially diagnostic clue exists. It further envisages performing positron emission tomography (PET) scan, if CT scan is unrevealing. Imaging of head and neck especially magnetic resonance imaging (MRI) has not been included in this algorithm, that leaves these important regions unexplored in most settings where PET scan is unavailable. MRI is a safe modality for evaluating central nervous system lesions and its role in FUO has not been adequately evaluated. We present three patients of FUO in whom the diagnosis of tuberculoma of brain as a cause of prolonged pyrexia got delayed because the MRI of head was not done initially, to comply with the approach of minimum diagnostic evaluation.
\end{abstract}

\section{Introduction}

Fever of unknown origin (FUO) is an important cause of morbidity and mortality in the world, especially in tropical and developing countries. It is defined as: i) temperature of $>101^{\circ} \mathrm{F}$ on several occasions; ii) a duration of fever of $>3$ weeks; iii) failure to reach a diagnosis after 3 outpatient visits or after 3 -days inpatient workup or after 1 week of intelligent and invasive ambulatory investigation. The most common causes of classic FUO are infection (30-40\%), malignancy (20\%-30\%), and collagen vascular disease (10\%-20\%); a diagnosis is not determined in $5 \%$ to $15 \%$ of cases. ${ }^{1}$ The burden of infectious causes lies largely in resource-starved regions of the world. Tu-

Correspondence: Khalid Javid Bhat, Department of Internal medicine, ASCOMS \& Hospitals, Jammu, Jammu \& Kashmir, 180017, India.

Tel.: +91.9469310056. E-mail: drkhalidjavid@gmail.com

Key words: Fever of unknown origin; magnetic resonance imaging; tuberculoma of brain.

Conflict of interest: the authors declare no potential conflict of interest.

Received for publication: 13 December 2014.

Revision received: 14 May 2015.

Accepted for publication: 14 May 2015.

This work is licensed under a Creative Commons Attribution NonCommercial 4.0 License (CC BY-NC 4.0).

CCopyright K.K. Pandita et al., 2016

Licensee PAGEPress, Italy

Italian Journal of Medicine 2016; 10:62-66

doi:10.4081/itjm.2015.575 berculosis (TB) is the most common infectious cause of FUO ( $41 \%$ of all infectious cases) in several studies from India. ${ }^{2}$ Moreover the diagnostic spectrum of FUO has changed over time due to recent advances in the laboratory diagnosis and imaging that have allowed for earlier diagnosis of these diseases. ${ }^{3}$ Magnetic resonance imaging (MRI) is a safe modality for evaluating central nervous system lesions and its role in FUO has not been adequately evaluated. We present three cases of tuberculoma of the brain manifesting itself as FUO in whom the cause of prolonged pyrexia was revealed by MRI of head. All patients gave written informed consent.

\section{Case Reports}

\section{Case \#1}

A 65-year-old Indian postmenopausal woman presented in July 2012 with complaints of low to moderate grade fever with chills, reduced appetite and lack of energy, for past two months. She had no history of cough, sputum production, jaundice, convulsions, joint pain or swelling, weight loss, bowel or urinary symptoms. She had no history of exposure to sick contacts. She had received several courses of empirical antibiotic therapy without any improvement. On examination she had fair general condition with oral temperature of $100.8^{\circ} \mathrm{F}$, pulse rate of 112 beats per min and blood pressure of 150/100 mmHg. Rest of her general and systemic examination was unremarkable. Laboratory investigations revealed hemoglobin of $11.7 \mathrm{~g} / \mathrm{dL}$, total leucocyte count of $5600 / \mathrm{mm}^{3}$, normal differential count, platelet count of $190,000 / \mathrm{mm}^{3}$ and erythrocyte sedimentation rate (ESR) of $40 \mathrm{~mm}$ at the end of $1^{\text {st }} \mathrm{h}$. Serum levels of bilirubin, aspartate transaminase, alanine transaminase, alkaline phosphatase, urea and creatinine were normal. 
She tested negative for malaria (OptiMAL-IT; TCS Biosciences Ltd., Buckingham, UK), HIV-1 and 2 [(enzyme-linked immunosorbent assay (ELISA)], hepatitis B surface antigen (HBsAg) and anti-hepatitis $\mathrm{C}$ virus $(\mathrm{HCV})$ antibody. Urine and blood cultures showed no growth and she tested negative for the anti-nuclear antibody (ANA). Chest X-ray was normal. Computed tomography (CT) scan of her chest, abdomen and pelvis was unrevealing and echocardiogram was normal. She was kept under close follow up. Two weeks later she had acute onset double vision with right-sided hemiparesis. There was no headache or neck stiffness. Contrast enhanced MRI of brain revealed multiple ring enhancing lesions with surrounding peri-lesional edema (Figure 1A). Serum and spinal fluid serology for neurocysticercosis was negative. We made a presumptive diagnosis of multiple tuberculomas of brain and started the administration of standard anti tubercular drugs (ATT) with oral prednisolone. During intensive phase of the treatment she became afebrile and her hemiparesis improved slowly but significantly, within four weeks. Oral steroids were tapered off and she continued ATT for one year. Follow up MRI brain after one year showed complete resolution of the lesions correlating with clinical disappearance of neurological deficit (Figure 1B).

\section{Case \#2}

A 26-year-old Indian pregnant woman presented in November 2012 with complaints of moderate grade re- mittent fever for the past month. She had no history of cough, vomiting, headache, seizures, joint pain, Raynaud's phenomenon, bladder or bowel symptoms. On examination she had mild pallor and oral temperature of $100.8^{\circ} \mathrm{F}$. Abdominal examination revealed fundal height corresponding to 34 weeks of pregnancy. Rest of her general and systemic examination was unremarkable. Routine laboratory investigations were grossly normal except for accelerated ESR of $102 \mathrm{~mm}$ at the end of $1^{\text {st }} \mathrm{h}$ and reduced hemoglobin of $9.8 \mathrm{~g} / \mathrm{dL}$. Urine and blood cultures were sterile and tests for malaria (OptiMAL-IT; TCS Biosciences Ltd.), HIV-1 and 2 (ELISA), HBsAg, anti-HCV antibody, ANA and QuantiFERON-TB Gold were unremarkable. Chest Xray was normal and ultrasound abdomen revealed single live fetus of 34 weeks of gestation. She had received injectable ceftriaxone therapy for one week, prior to presentation without any improvement. We made the diagnosis of FUO with no potentially diagnostic clue and followed her on outpatient basis. She presented again one week after delivery in the first week of January 2013 with acute behavioral disturbance of one-day duration. She was agitated and speaking incoherently and did not allow physical examination. She had no history of any previous psychiatric illness. We gave her one intramuscular haloperidol $5 \mathrm{mg}$ for possible postpartum psychosis and her agitation abated. On examination, she had mild pallor and oral temperature of $100.2^{\circ} \mathrm{F}$ and a solitary, non-tender cervical lymph node was palpable on right

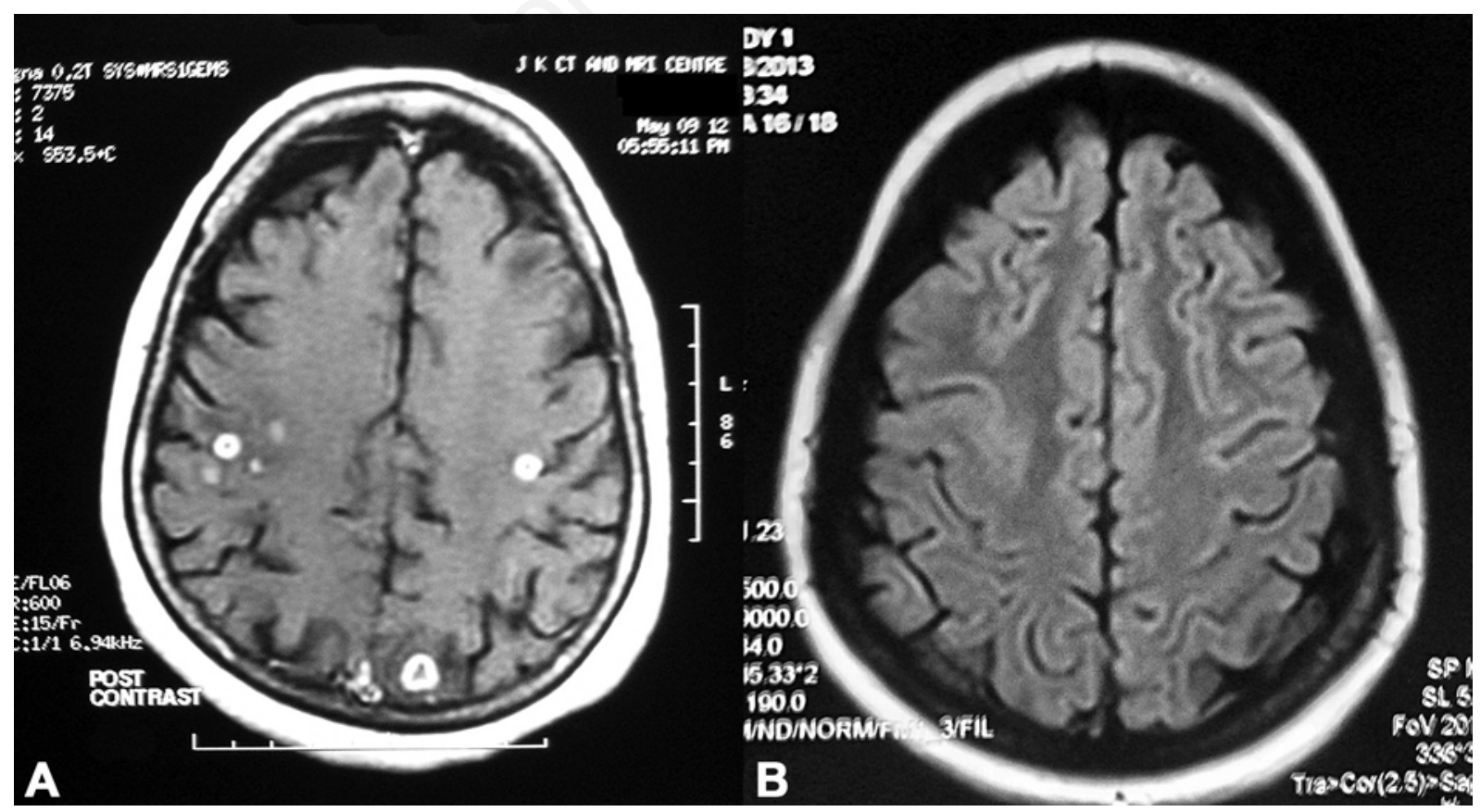

Figure 1. A) Magnetic resonance imaging (MRI) contrast showing multiple cerebral ring enhancing lesions before starting standard anti tubercular drugs (ATT). B) Follow up MRI showing clearing of tuberculomas on ATT. 
side. Rest of the examination was unremarkable except for palpable involuting uterus. Repeated laboratory investigations were all non-contributory. In view of her behavioral disturbance, contrast enhanced MRI of brain was done which showed multiple cerebral lesions with perilesional edema (Figure 2A). Neurocysticercosis serology was negative. With a high clinical suspicion of tuberculomas, the patient was put on standard ATT along with oral steroids. In the meantime histopathology report of excised lymph node showed caseating granuloma with multiple giant cells suggestive of tuberculosis. She became afebrile after two weeks and a follow up MRI done seven months later showed complete resolution of the lesions (Figure 2B).

\section{Case \#3}

A 63-year-old Indian man was referred from community hospital in May 2013 with complaints of fever and fatigue for the past month. He had been evaluated there for FUO and had received oral antibiotics as an empirical therapy. On examination, he was mildly anemic, with oral temperature of $100^{\circ} \mathrm{F}$, pulse rate of 110 beats per min and blood pressure of 110/90 $\mathrm{mmHg}$. Laboratory investigations revealed hemoglobin of 11.7 $\mathrm{g} / \mathrm{dL}$, total leucocyte count of $6600 / \mathrm{mm}^{3}$, normal differential count, and ESR of $40 \mathrm{~mm}$ at the end of $1^{\text {st }} \mathrm{h}$. His renal and liver function tests were normal and ANA screening was negative. Blood urine and sputum cultures were sterile. CT scan of his chest, abdomen including pelvis was normal and he was kept under close follow up. Two weeks later he started complaining of subtle difficulty in recalling names and dates. His neurological examination was otherwise normal. Repeat laboratory investigations and metabolic parameters were normal. With the view of evaluating him for cognitive changes, contrast enhanced MRI of brain was done which revealed multiple ring enhancing lesions consistent with tuberculomas of brain (Figure 3). We started the administation of standard ATT and oral prednisolone. His fever subsided after two weeks of ATT therapy and there was no further worsening of his cognition. Follow up MRI brain after 8 months showed complete resolution of the lesions.

\section{Discussion and Conclusions}

Prolonged pyrexia is more commonly an atypical manifestation of a more common illness rather than a manifestation of an exotic disease. TB of the central nervous system (CNS) accounts for $10 \%$ of extra pulmonary cases of or $0.7 \%$ of all TB cases. ${ }^{4}$ In the developing world, tuberculoma is the second commonest manifestation of CNS tuberculosis and accounts for around $20-30 \%$ of space-occupying lesions in the brain. ${ }^{5}$ They can be silent and at times manifestations can be non-specific because of their size, lack of significant edema and strategic parenchymal location and hence pose a clinical challenge. However being a treatable condition, early diagnosis helps to prevent morbidity and mortality. Unlike tubercular meningitis, which has a vivid presentation, tuberculoma of the brain has an insidious course. It is a major neurological problem in developing countries. Constitutional signs and symptoms commonly associated with TB infection are present in less than one third of patients with tuberculoma of the brain and pyrexia is reported in only $20-25 \%$ of cases. ${ }^{6}$ In the literature most of the cases of brain tuberculomas have been reported from

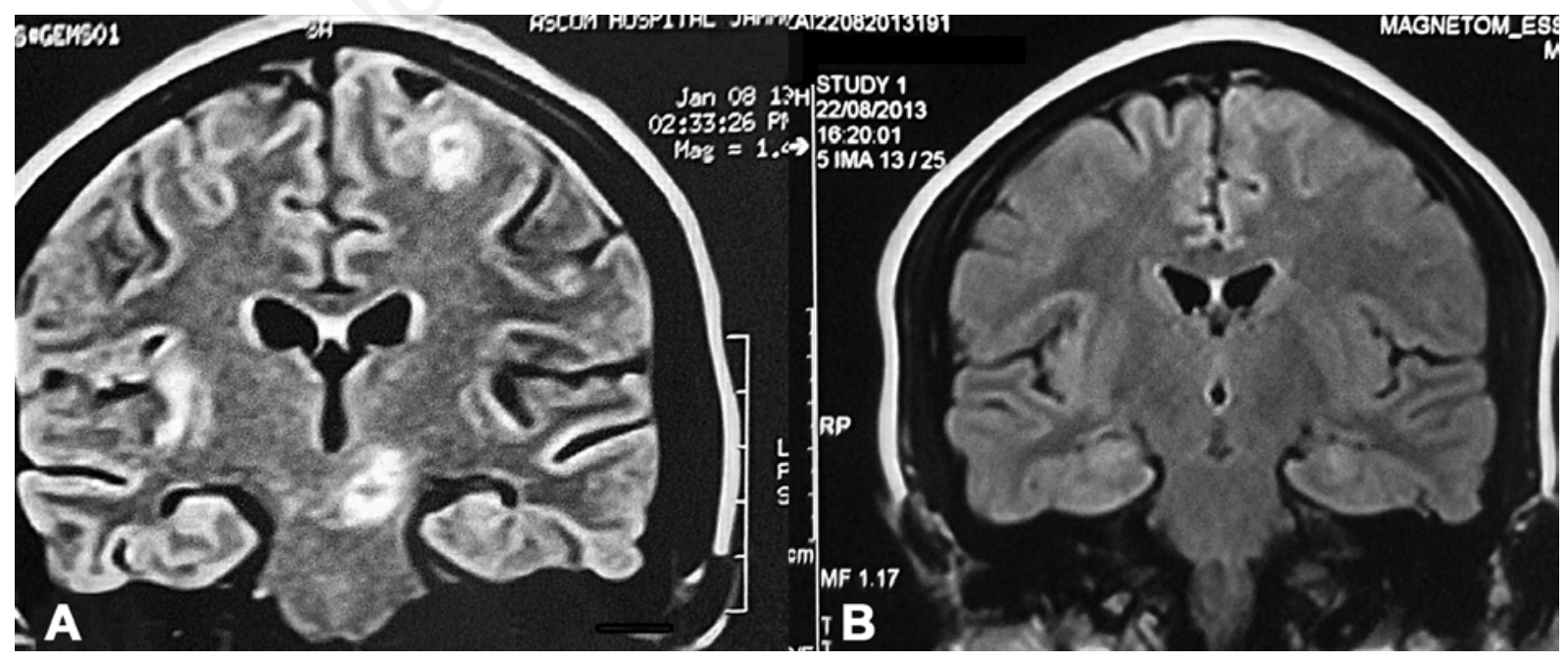

Figure 2. A) Magnetic resonance imaging (MRI) scan (coronal view) showing multiple tuberculomas with perilesional edema. B) Follow up MRI showing resolutions of tuberculomas on standard anti tubercular drugs. 
India, France, Mexico, China and Turkey. Most of the patients presented with seizures, focal neurological deficits and headache along with systemic symptoms and signs. However FUO as a sole presentation was rarely seen. ${ }^{7}$

The diagnosis of tuberculoma of brain is made in relation to the clinical, epidemiological and radiographic features or by tissue biopsy of the lesion. MRI scan of brain with non-caseating tuberculoma appears as hyperintense lesions with ring shaped contrast enhancement on T2 images, and the granulomas with solid caseation appear hypointensite on T2 weighted images. Tuberculomas with central liquefaction appear hyperintense with a central hypointensity on T2 weighted images. The common differential diagnosis includes neurocysticercosis, malignant disease, sarcoidosis, pyogenic abscess and toxoplasmosis. ${ }^{8}$ Although stereotactic biopsy is essential but in resource poor settings, it is usually avoided or unavailable.

The goals of workup of a patient of FUO are to find out the pathology, organ/s involvement and the causative organism in case of infective pathology. In our patients these goals were reasonably accomplished by a single investigation modality viz MRI scan of the brain. MRI scan of brain was requested only when there were clear cut clues to the possible

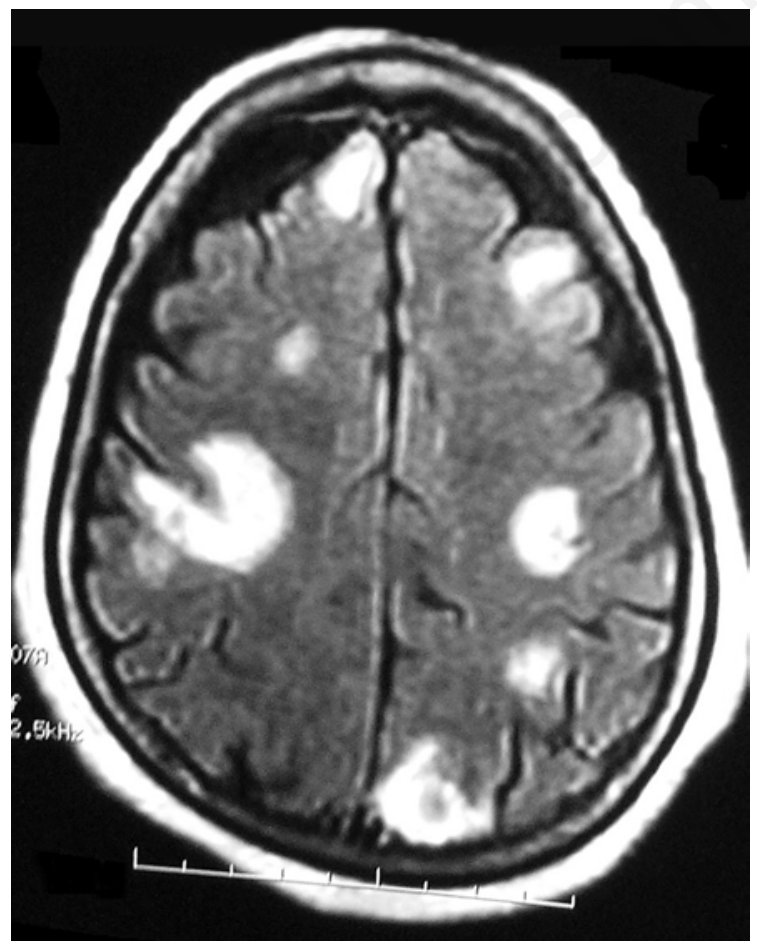

Figure 3. Magnetic resonance imaging contrast showing multiple cerebral ring enhancing lesions before treatment. involvement of brain in the form of new signs and symptoms e.g., hemiparesis in one patient and behavioral disturbance and cognitive changes in the other two respectively. This was long after the patient's family members and health care providers had to pass through much anguish and frustration as is usual in the investigation of FUO.

The stepwise algorithm for the workup of FUO patients envisaged by de Kleijn and colleagues broadly divides the patients of FUO in two arms viz those with potential diagnostic clues and those with no potential diagnostic clues. ${ }^{9}$ The algorithm suggests to perform $\mathrm{CT}$ of chest, abdomen and pelvis in the latter category and whole body Fluorodeoxyglucose F18 (FDG) positron emission tomography (PET) scan in case the results of imaging scan are negative..$^{10}$ For many weeks before the above patients presented with potential diagnostic clues they belonged to the latter arm of algorithm. In patient number one and three, CT scan of chest and abdomen was unremarkable and in patient number two, it had been deferred in view of pregnancy. Whole body FDG PET scan is not available in this part of the country, so virtually health care providers were following up the three patients till the clues appeared in them. If we had done MRI of the brain at the earlier stage probably we would have arrived at the diagnosis much earlier. In addition MRI is a safe modality in pregnancy and is cost effective as compared to investigations like FDG PET, gallium scan, Tc-99 Leukoscan. ${ }^{11}$ Although comparative utility MRI and CT in the diagnosis of FUO is unknown, MRI, in general, is better than CT scan for detecting lesions of nervous system. ${ }^{12}$ FDG PET scanning is superior to other forms of nuclear scanning. FDG used in PET scan demonstrates the site of pathology and directed histologic diagnosis as it accumulates in tumors and sites of inflammation. However its use in the diagnosis of FUO is limited by its high cost and wide unavailability. ${ }^{13}$

The role of the radiologic techniques in FUO is to localize the potential cause of the fever considering the as low as reasonably achievable principle and the cost of modern diagnostic examinations. ${ }^{14} \mathrm{MRI}$, an encouraging modality has been used for a narrow range of indications during evaluation of FUO especially when systemic vasculitis is suspected as a cause of prolonged pyrexia. ${ }^{15} \mathrm{We}$ believe that these case reports will stimulate further research about the role of MRI in evaluation of patients of FUO especially in the developing countries where infection is still the most common and important cause of FUO. We suggest that patients of FUO with no potential diagnostic clue in whom CT scan of chest, abdomen, and pelvis is unrevealing, should undergo MRI scan of head, till PET scan becomes widely available as FUO itself may be hidden in the head. 


\section{References}

1. Hirschmann JV. Fever of unknown origin in adults. Clin Infect Dis 1997;24:291-302.

2. Bandyopadhyay D, Bandyopadhyay R, Paul R, Roy D. Etiological study of fever of unknown origin in patients admitted to medicine ward of a teaching hospital of Eastern India. J Glob Infect Dis 2011;3:329-33.

3. Tabak F, Mert A, Celik AD, et al. Fever of unknown origin in Turkey. Infection 2003;31:417-20.

4. Dye C, Scheele S, Dolin P, et al. Global burden of tuberculosis - estimated incidence, prevalence, and mortality by country. JAMA 1999;282:677-86.

5. Garg RK. Diagnosis of intracranial tuberculoma. Ind J Tub 1996;43:35-9.

6. Modi M, Garg A. Tuberculosis of central nervous system. J Postgrad Med Edu Res 2013;47:202-13.

7. Psimaras D, Bonnet C, Heinzmann A, et al. Solitary tuberculous brain lesions: 24 new cases and a review of literature. Rev Neurol 2014;170:454-63.

8. Gupta RK, Pandey R, Khan EM, et al. Intracranial tuberculomas: MRI signal correlation with on histopathology and localized proton MR spectroscopy. Magn Reson Imag 1991:2:443-9.
9. de Kleijn EM, van der Meer JW. Inquiry into the diagnostic workup of patients with fever of unknown origin. Neth J Med 1997;50:69-74.

10. Bleeker-Rovers CP, Vos FJ, de Kleijn EM, et al. A prospective multicenter study on fever of unknown origin: the yield of a structured diagnostic protocol. Medicine (Baltimore) 2007;86:26-38.

11. Kanal E, Barkovich AJ, Bell C, et al. ACR Blue Ribbon Panel on MR Safety. ACR guidance document for safe MR practices. AJR Am J Roentgenol 2007;188:1447-74.

12. Laughlin S, Montanera W. Central nervous system imaging. When is CT more appropriate than MRI? Postgrad Med 1998;104:73-91.

13. Gelfand JA, Callahan MV. Fever of unknown origin. In: Longo DL, Fauci AS, Kasper DL, et al., eds. Harrison's principles of internal medicine. $18^{\text {th }} \mathrm{ed}$. vol 2. New York: McGraw Hill; 2012. pp 158-164.

14. Kaya A, Ergul N, Kaya SY, et al. The management and the diagnosis of fever of unknown origin. Expert Rev Anti Infect Ther 2013;11:805-15.

15. Wagner AD, Andresen J, Raum E, et al. Standardised work-up programme for fever of unknown origin and contribution of magnetic resonance imaging for the diagnosis of hidden systemic vasculitis. Ann Rheum Dis 2005;64:105-10. 